\title{
Comparative study of computed tomography of normal and lymphoid follicular hyperplasia thymus in myasthenia gravis patients
}

\author{
HUI ZHANG ${ }^{1}$, PENG ZHANG ${ }^{1}$ and TIE-LIAN YU ${ }^{2}$ \\ Departments of ${ }^{1}$ Cardiac and Thoracic Surgery and ${ }^{2}$ Radiology, \\ Affiliated General Hospital of Tianjin Medical University, Tianjin 300051, P.R. China
}

Received March 20, 2018; Accepted August 18, 2018

DOI: $10.3892 /$ etm.2018.6948

\begin{abstract}
The aim of the present study was to evaluate the thymuses of non-thymomatous myasthenia gravis (MG) patients by computed tomography (CT) for differentiating lymphoid follicular hyperplasia (LFH) thymus from normal/involuted thymus in order to assist surgeons in determining whether a non-thymomatous MG patient requires an operation. In the present retrospective review over 10 years, 80 patients who received CT scan and thymectomy at the Affiliated General Hospital of Tianjin Medial University (Tianjin, China) were included. According to the pathological records, 54 of the cases initially detected on CT were confirmed as LFH thymus. Thymic measurements, including anteroposterior and transverse dimensions, width (the longest axis of the lobe on a transverse scan) and thickness (the largest dimension perpendicular to the long axis of the lobe) and CT attenuation of the thymus region, adipose tissue and chest wall musculature in each CT slice were included to assess differences between the LFH group and the normal/involuted thymus group. Although a negative association between patient age and the CT attenuation of the thymus region was identified $(\mathrm{r}=-0.779, \mathrm{P}<0.05$, Pearson's correlation test), the LFH thymus group featured nodular changes on CT, while no such changes were observed in the normal/involuted thymus group. The mean age of disease onset in the LFH thymus group was significantly lower than that in the normal thymus group $(40.2 \pm 17.3$ vs. $59.2 \pm 9.3$ years $)$. Furthermore, significant differences in CT attenuation were identified between the LFH group and the normal/involuted thymus group [-41.21 \pm 54.42 vs. $-108.23 \pm 8.72$ Hounsfield units (HU) on unenhanced CT; $-25.57 \pm 58.65$ vs.-117.40 $\pm 6.22 \mathrm{HU}$ on contrast-enhanced CT]. In the LFH group, the difference in
\end{abstract}

Correspondence to: Professor Peng Zhang, Department of Cardiac and Thoracic Surgery, Affiliated General Hospital of Tianjin Medical University, 154 Anshan Road, Tianjin 300051, P.R. China E-mail: zhp_tjgh@yahoo.com.cn

Key words: thymus gland, lymphoid follicular hyperplasia, involuted thymus, myasthenia gravis, computed tomography, thymectomy mean CT attenuation between the thymus region and adipose tissue was significant, while no significant difference was observed in the normal/involuted thymus group. In conclusion, CT may be used to distinguish LFH thymus from normal/involuted thymus in MG patients.

\section{Introduction}

Myasthenia gravis (MG) is an autoimmune disease, which is caused by anti-nicotinic acetylcholine receptor antibody, resulting in a defect in neuromuscular transmission (1). Clinically, MG is manifested by weakness of voluntary muscles on prolonged exercise, which is restored rapidly after rest (1). Although the trigger of autoimmunity in MG is unknown, it is well documented that the thymus has an important role in the pathogenesis of MG $(2,3)$. An estimated $80-90 \%$ of MG patients have thymic abnormalities, of which lymphoid follicular hyperplasia (LFH) accounts for $65-75 \%$ (4). Thymectomy has almost always been routinely performed for patients with MG and is effective in most cases of LFH (5-8). From the position of the thoracic surgeon and under consideration of the cost, pre-operative computed tomography (CT) is a more valuable method than magnetic resonance imaging and ${ }^{201}$ Tl-single photon emission CT in diagnosing the abnormalities of thymus, including LFH and thymoma. However, CT has its own limitations in differentiating LFH from normal and involuted thymus considering the radiology standards for thymus LFH (9-13). Whether a patient with non-thymomatous MG requires a thymectomy is currently difficult to decide for the surgeon. As described previously, the overall volume of the thymus remains stable after birth and the volume of the thymus epithelial space diminishes during aging, while thymopoiesis is encountered in thymus tissues of normal individuals aged $>60$ years $(14,15)$. However, most patients with $\mathrm{MG}$ have an LFH thymus, which is enlarged and contains B-cell germinal centers $(4,16,17)$. The present study assessed the chest CT of MG patients who received thymectomy after one week following admission. The removed thymuses were then confirmed as either normal/involuted or LFH by pathological analysis. On the basis of the pathological results, a comparison of the CT appearance between the LFH thymus group and the normal/involuted thymus group was performed in order to 
determine the best indicators of thymus abnormalities of LFH for the thoracic surgeon.

\section{Materials and methods}

Patients and thymuses. Of the $80 \mathrm{MG}$ patients included, 54 were diagnosed as having LFH thymuses (21 males and 33 females; age range, $11-73$ years; mean age, $40.2 \pm 17.28$ years). The other 26 patients were diagnosed by the pathologist as having normal or involuted thymuses (20 males and 6 females; age range, 42-72 years; mean age, $58.57 \pm 8.21$ years). All of the patients received transsternal thymectomy between January 2001 and January 2011 at the Affiliated General Hospital of Tianjin Medical University (Tianjin, China). Written informed consent was obtained from all of the patients who participated and the Ethics Committee of the Affiliated General Hospital of Tianjin Medical University (Tianjin, China) approved the study protocols.

The diagnosis of MG was made based on the patients' history, the results of neurological examinations, electrophysiological studies (repetitive nerve stimulation, single fiber electromyography) and a prostigmin test. The patients did not receive any immunosuppressants, including corticosteroids, prior to the operation. Patients with other diagnoses, including Grave's disease, malignant tumors receiving chemotherapy, polyneuropathy, myopathy, multiple sclerosis or neurasthenic syndrome, were excluded.

The pathological diagnoses were made by a senior pathologist without any information from imaging and clinical studies. There are two histopathological diagnoses for the thymus in MG: LFH and normal/involuted thymus. Hyperplasia is characterized by the appearance of lymphoid follicles, which are usually round-shaped accumulations of B lymphocytes with or without germinal centers on microscopic examination. Normal thymus was considered when no lymphoid follicles were present in the perivascular space with or without thymic atrophy or adipose tissue alone (18).

Spiral CT. Spiral CT images were acquired on a single scanner with contiguous 1.3-10.0 mm thick slices using a whole-body CT (GE Lightspeed 64; GE Healthcare, Little Chalfont, UK) in the mediastinal field condition. Standard parameters for spiral CT of the chest were $120 \mathrm{kVp}$ and 200-340 mAsec. The images were reconstructed with a standard soft-tissue-kernel algorithm. Contrast-enhanced CT scans were obtained after intravenous injection of 120-150 ml contrast material (iohexol; $140 \mathrm{mg} / \mathrm{ml}$; GE Healthcare) by using a power injector at a rate of 2.0-4.0 ml/sec. The injection volume and rate of contrast medium delivery varied depending on the patient's weight and vascular access. Radiological findings were determined by a senior radiologist prior surgery.

Analysis of CT images. CT images were retrieved from the institutional picture archiving and communication system and analyzed on a clinical workstation (GE Healthcare). The upper border of the mediastinal tissue was fixed at the level of the aortic arch and the lower border was fixed at the junction of the pulmonary artery and the heart (19).

According to St Amour et al (20) and Francis et al (21), the shape (quadrilateral or triangular) and density of the thymus were determined in all patients. The use of the terms triangular and quadrilateral was chosen for simplicity and conformity with prior studies (22). Thymic measurements, including anteroposterior (AP) and transverse dimensions, width (the longest axis of the lobe on a transverse scan) and thickness (the largest dimension perpendicular to the long axis of the lobe), were also obtained for each of the patients (20). The craniocaudal dimension of the thymus was not determined, since precise measurement was usually not possible due to variations in the degree of inspiration on contiguous CT scan (20).

The pixels of the thymus region, muscle and adipose tissue in the chest wall in the same CT slice were measured using the workstation to compare the pixel attenuation of different tissues. The mean CT attenuation of each region in each group of patients (LFH and normal thymus) was compared, by studying differences in the tissues within each group and between the groups of patients for the same tissue.

Statistical analysis. Values are expressed as the mean \pm standard deviation unless otherwise specified. The Kolmogorov-Smirnov test was used to test if the quantitative data had a normal distribution. The independent-samples t-test was used to examine the differences in thymic thickness, AP dimension and perpendicular dimension between the two MG groups. Analysis of variance followed by the Student-Neuman-Keuls post-hoc test was used to examine differences in mean $\mathrm{CT}$ attenuation among thymus tissue, fat tissue and muscle in the two groups. Pearson's rank correlation was used to test the association between the patients' age and the CT attenuation. Analyses were performed using SPSS 13.0 (SPSS, Inc., Chicago, IL, USA). P<0.05 was considered to indicate a statistically significant difference.

\section{Results}

CT dimensions of the thymus region. First, when all of the patients were analyzed as a whole and not grouped according to their histopathological results, a negative correlation between the patients' age and the CT attenuation in Hounsfield units (HU) of the thymus region was identified ( $\mathrm{r}=-0.779 ; \mathrm{P}<0.05$; Pearson's rank correlation test; Fig. 1), which means the that the CT attenuation in $\mathrm{HU}$ of the thymus region of MG patients decreases with age. The mean age at disease onset in the LFH thymus group was lower than that in the normal thymus group $(40.2 \pm 17.3$ vs. $59.2 \pm 9.3$ years; $\mathrm{P}<0.001$; Table I). In the LFH group, the thymus or its remnant was detected in all of the MG patients. All of the thymuses remained in their usual triangular or quadrilateral cross-sectional shape and the two types of shape were observed in patients in each decade of life. Even in patients in their fifties, it was possible to distinguish the thymus region from adipose tissue. However, in the cases where the thymus had been replaced by adipose tissue, certain nodules were present in the thymus region, which did not occur in normal thymus of MG patients. Typically, the gland was located anterior to the ascending aorta, pulmonary outflow tract and distal superior vena cava within the anterior mediastinal fat. Among the MG patients with LFH (Figs. 2-9), no case of aberrantly positioned thymus was encountered. On the other hand, in the MG patients of the normal thymus group, it was not possible to detect the thymus or its remnant, as the thymus tissue had been completely infiltrated by adipose tissue. In all 
Table I. Clinicopathological features of the patients.

\begin{tabular}{lcc}
\hline Parameter & LFH thymus group $(\mathrm{n}=54)$ & Normal/involuted thymus group (n=26) \\
\hline Gender & & \\
Male & $21(26.3 \%)$ & $20(25.0 \%)$ \\
Female & $33(41.3 \%)$ & $6(7.5 \%)$ \\
Age of disease onset (years) & $11-73$ & $40-72$ \\
Range & $40.2 \pm 17.3$ & $59.2 \pm 9.3$ \\
Mean \pm standard deviation & & $<0.001$ \\
\hline
\end{tabular}

LFH, lymphoid follicular hyperplasia.

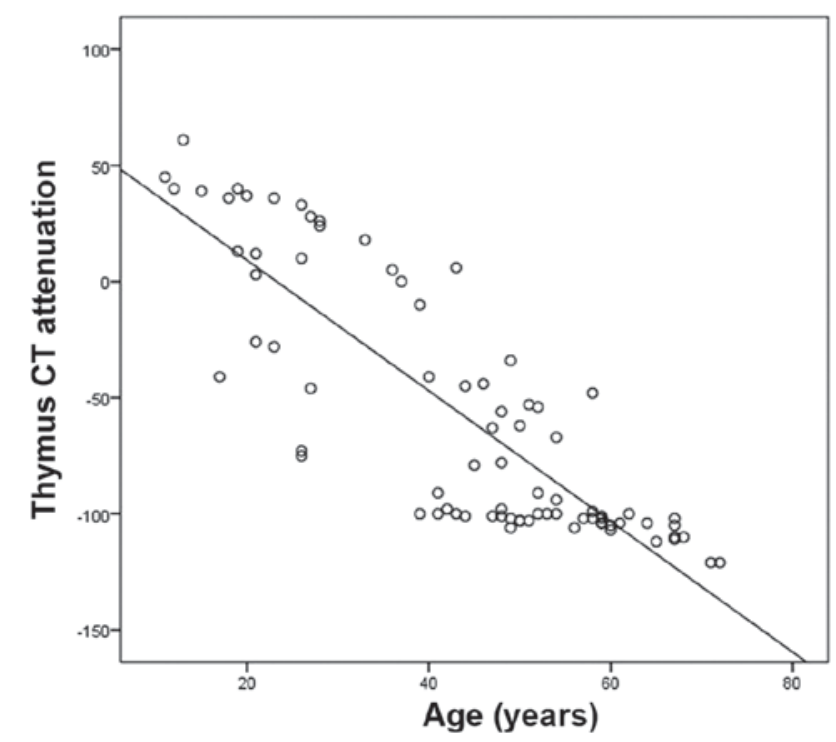

Figure 1. Correlation of the thymus CT attenuation with the age of the patients $(\mathrm{r}=-0.779 ; \mathrm{P}<0.05)$. $\mathrm{CT}$, computed tomography.

MG patients with normal/involute thymus, the shape of the thymus region on CT was quadrilateral (Figs. 10-12), which may be due to the patients' age or a different pathogenesis from that in the LFH MG group.

Regarding the mean measures of thymic thickness, width, AP dimension and transverse dimension, there were no statistical differences between the LFH group and normal/involuted thymus group (Table II).

CT attenuation of thymus region on unenhanced $C T$. A total of 43 and 18 patients were subjected to unenhanced CT in the LFH group and the normal/involuted thymus group, respectively. The mean values of $\mathrm{CT}$ attenuation of different regions are listed in Table III. In the LFH group, when comparing the $\mathrm{CT}$ attenuation of different regions (thymus region vs. adipose tissue, thymus region vs. muscle tissue, adipose tissue vs. muscle tissue), the differences were all significant $(\mathrm{P}<0.05)$. In the normal/involuted thymus group, there was no significant difference in the mean CT attenuation between thymus region and adipose tissue, while the differences were significant between other tissues (thymus region vs. muscle tissue, adipose tissue vs. muscle tissue,
$\mathrm{P}<0.05)$. Most importantly, there was a significant difference in the CT attenuation of the thymic region between the two groups (Table III).

CT attenuation of thymus region on contrast-enhanced CT. In the LFH group and the normal/involuted thymus group, 11 and 8 patients received contrast-enhanced CT, respectively. The mean values of CT attenuation of different regions are presented in Table IV. Similar to the trends of the unenhanced CT, a significant difference in the CT attenuation of the thymic region was identified between the two groups (Table IV). Within each group, when comparing the CT attenuation of different regions (thymus region vs. adipose tissue, thymus region vs. muscle tissue, adipose tissue vs. muscle tissue), the differences were all significant $(\mathrm{P}<0.05)$.

\section{Discussion}

In general, the thymus in a healthy human subject is a central lymphoid organ that is gradually replaced by adipose tissue, which results in thymic involution. The processes/timing of the weight and volume of the thymus reaching its apex and the beginning of involution are inconsistently described among previous studies (19-21). However, it is known that when the involution starts, the epithelial component of the thymus atrophies, resulting in scattered small lymphocytes in abundant adipose tissue $(18,19,22)$. LFH, also known as autoimmune thymitis, is characterized by a normal size and weight of the thymus with chronic inflammation and proliferation of lymphoid follicles, active germinal centers and increased numbers of lymphocytes and epithelial cells $(23,24)$. The symptoms of MG, including the impairment of ocular (extra-ocular muscles, eyelids), bulbar (ingestion function, voice/speech function, respiratory function, facial muscles) and limb-axial muscles (arms, legs and neck), always improve after thymectomy in patients with thymoma or LFH thymus (5-8). Furthermore, in MG patients with $\mathrm{LFH}$, the thymus also frequently appears as atrophic on CT scan and gross examination, particularly in elderly patients. Differentiation of LFH from the normal thymus may be difficult for the radiologist on the basis of morphologic features alone. These all present obstacles for the thoracic surgeon when determining whether an MG patient requires a thymectomy. 


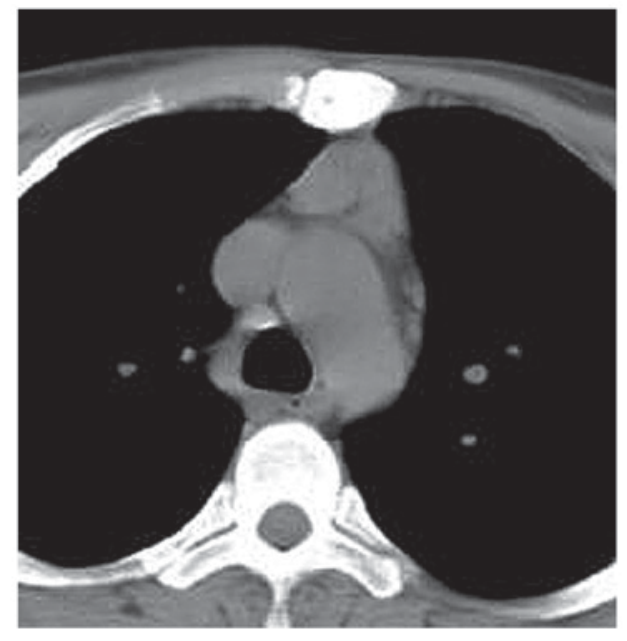

Figure 2. Unenhanced computed tomography image of a male myasthenia gravis patient (age, 18 years) with lymphoid follicular hyperplasia. The thymus is triangular in shape and may be easily distinguished from adjacent tissue.

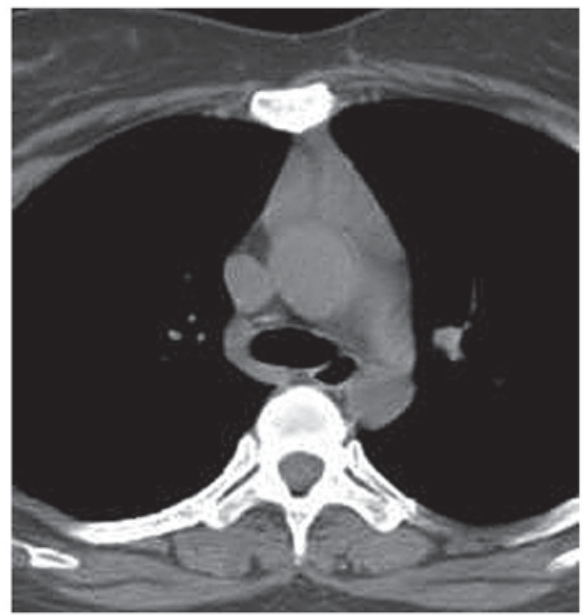

Figure 3. Female myasthenia gravis patient (age, 26 years) with lymphoid follicular hyperplasia. The septum between the two lobes is visible.

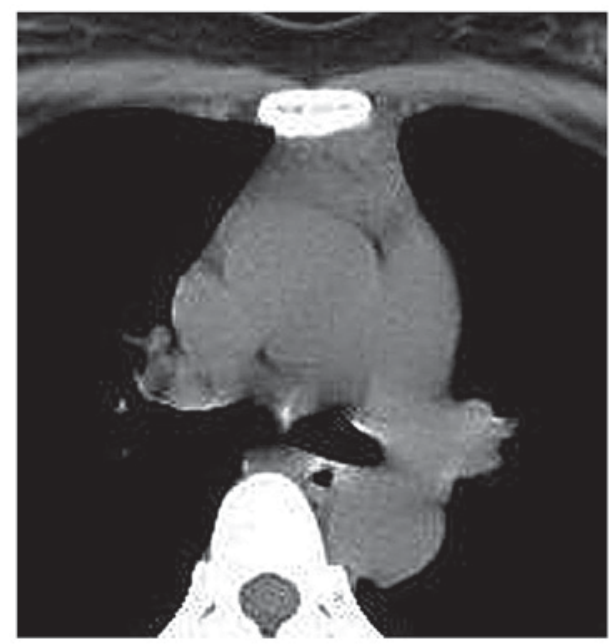

Figure 4. Female myasthenia gravis patient (age, 36 years) with lymphoid follicular hyperplasia. The thymus is quadrilateral and the computed tomography attenuation in the thymus region is different from that of the adipose tissue and musculature.

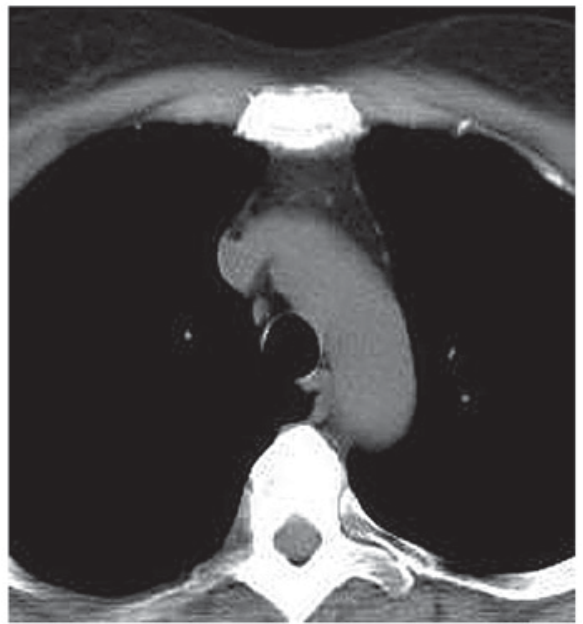

Figure 5. Female myasthenia gravis patient (age, 45 years) with lymphoid follicular hyperplasia. The thymus is quadrilateral in shape and nodules are visible in the fat-infiltrated thymus region.

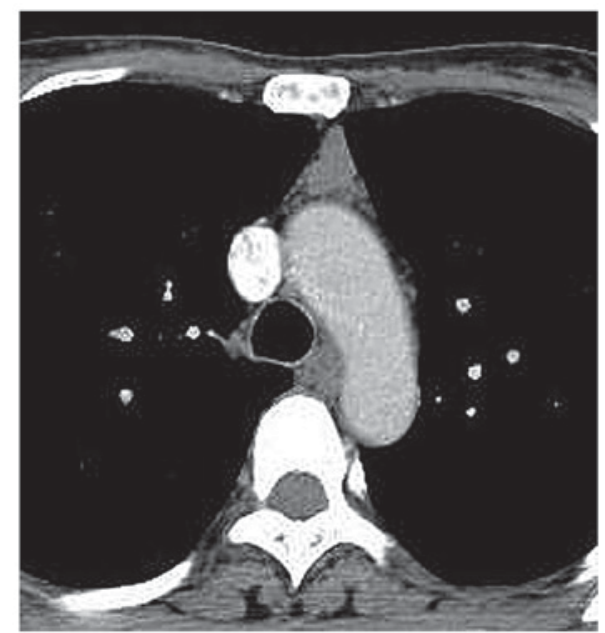

Figure 6. Contrast-enhanced computed tomography image of a female myasthenia gravis patient (age, 50 years) with lymphoid follicular hyperplasia. The thymus is triangular in shape and can be easily distinguished from adjacent tissue, particularly adipose tissue.

In the present study, the data of CT scans of non-thymomatous MG patients with LFH thymus or normal/involuted thymus, which had been pathologically confirmed, were analyzed. The results indicated that the mean age of disease onset in the LFH thymus group was lower than that in the normal/involuted thymus group $(40.2 \pm 17.28$ vs. $58.57 \pm 8.21$ years $)$. In the LFH group, the age was distributed more widely (from 11 to 73 years) than in the normal/involuted thymus group (42-72 years). This result may possibly indicate a different mechanism between younger and older MG patients (25). In fact, the observation that there were no significant differences between the two groups in six views of the thymus exactly coincides with the fact that the autoimmune LFH thymus is characterized by a normal size and weight, including those of lymphoid follicles (24).

In the present study, the CT attenuation of the thymus region of MG patients gradually decreased with age, although for the spiral CT mentioned above, the parameters diversified. 


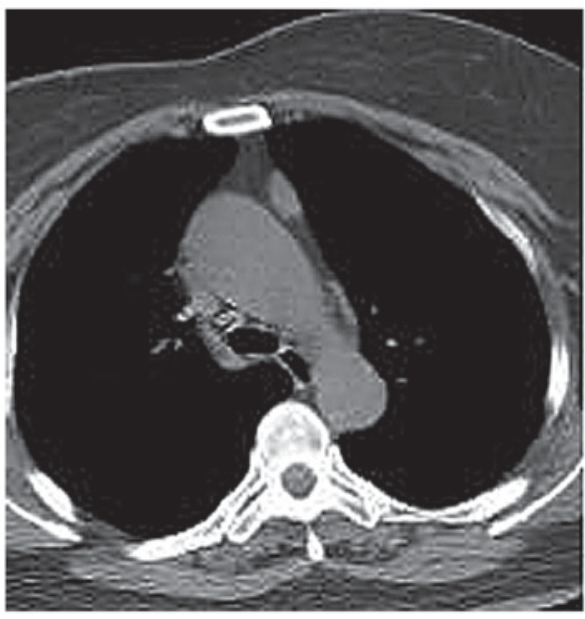

Figure 7. Female myasthenia gravis patient (age, 54 years) with LFH. The thymus is triangular in shape and a nodule is apparent, which was later pathologically confirmed as LFH. LFH, lymphoid follicular hyperplasia.

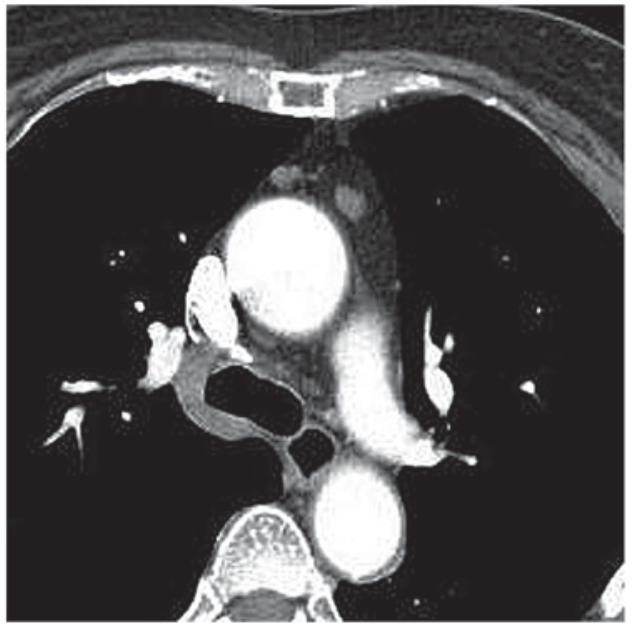

Figure 8. Contrast-enhanced computed tomography image of a female myasthenia gravis patient (age, 58 years) with lymphoid follicular hyperplasia. The thymus is triangular in shape and nodules are apparent in the thymus region.

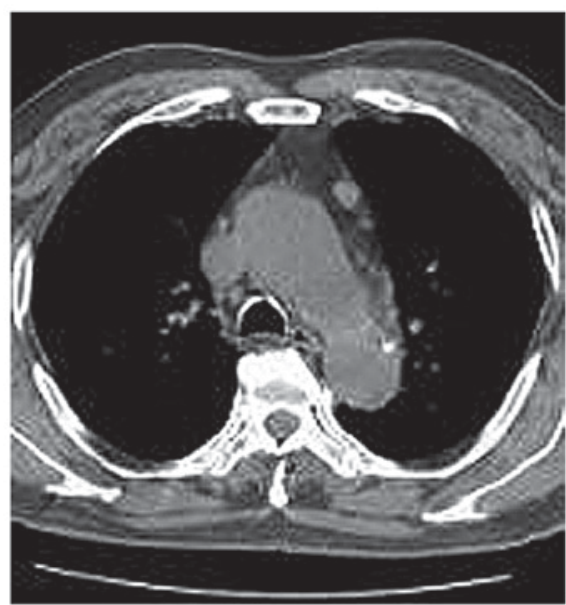

Figure 9. Unenhanced computed tomography image of a male patient (age, 67 years) with myasthenia gravis and lymphoid follicular hyperplasia The thymus is quadrilateral in shape and certain nodules may be distinguished from adipose tissue.

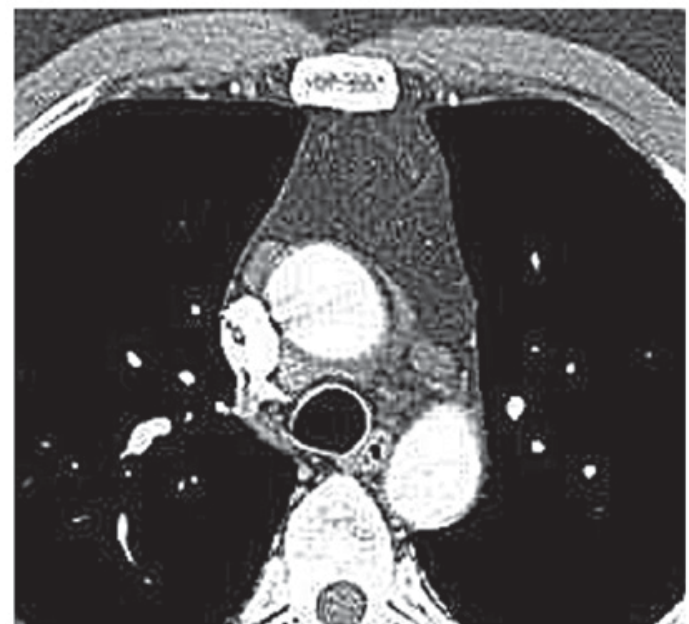

Figure 10. Contrast-enhanced CT image of a male myasthenia gravis patient (age, 43 years) with normal thymus. The thymus is quadrilateral in shape and homogeneous regarding $\mathrm{CT}$ attenuation. CT, computed tomography.

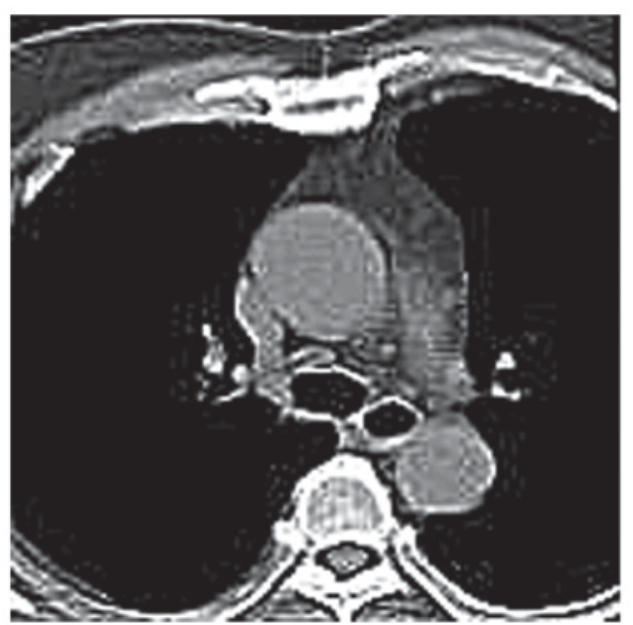

Figure 11. Unenhanced CT image of a male myasthenia gravis patient (age, 59 years) with normal (involuted) thymus. The thymus is quadrilateral in shape and homogeneous regarding CT attenuation. CT, computed tomography.

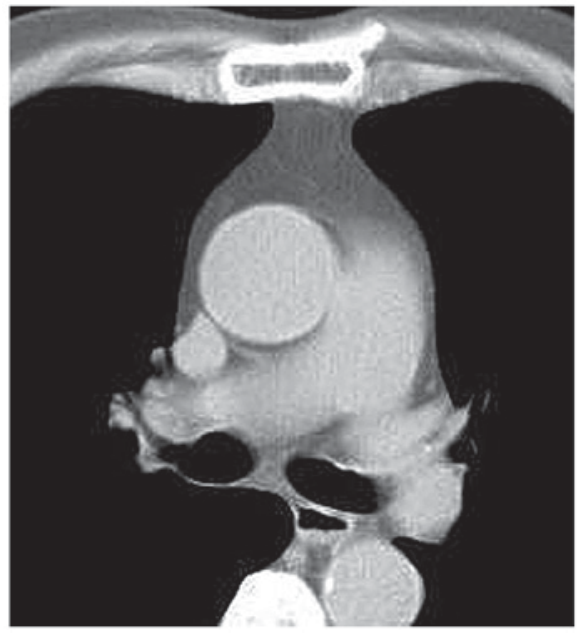

Figure 12. Unenhanced CT image of a male myasthenia gravis patient (age, 72 years) with normal thymus. The thymus is quadrilateral in shape and homogeneous regarding $\mathrm{CT}$ attenuation. $\mathrm{CT}$, computed tomography. 
Table II. Thymic measurements of myasthenia gravis patients with LFH or normal/involuted thymus (cm).

\begin{tabular}{lccc}
\hline Type of measurement & LFH thymus group $(\mathrm{n}=54)$ & Normal/involuted thymus group (n=26) & P-value \\
\hline Thickness & $2.03 \pm 0.71$ & $2.57 \pm 0.65$ & 0.41 \\
Width & $3.70 \pm 1.37$ & $4.48 \pm 2.29$ & 0.22 \\
AP dimension & $2.48 \pm 0.99$ & $2.42 \pm 0.75$ & 0.86 \\
Transverse dimension & $4.76 \pm 1.54$ & $5.76 \pm 1.26$ & 0.16 \\
\hline
\end{tabular}

Values are expressed as the mean \pm standard deviation. LFH, lymphoid follicular hyperplasia; AP, anteroposterior.

Table III. Unenhanced computed tomography attenuation of different types of soft tissue in non-thymomatous myasthenia gravis patients.

\begin{tabular}{lccc}
\hline CT attenuation $(H U)$ & LFH thymus group $(\mathrm{n}=43)$ & Normal/involuted thymus group $(\mathrm{n}=18)$ & P-value \\
\hline Thymus region & $-41.21 \pm 54.42$ & $-108.13 \pm 8.7$ & 0.02 \\
Adipose tissue & $-107.61 \pm 14.16^{\mathrm{a}}$ & $-110.24 \pm 4.17$ & 0.72 \\
Muscle tissue & $53.62 \pm 19.83^{\mathrm{a}, \mathrm{b}}$ & $48.5 \pm 17.94^{\mathrm{a}, \mathrm{b}}$ & 0.62 \\
\hline
\end{tabular}

${ }^{\mathrm{a}} \mathrm{P}<0.001$ vs. thymus region in the same group; ${ }^{\mathrm{b}} \mathrm{P}<0.001$ vs. adipose tissue in the same group. Values are expressed as the mean \pm standard deviation. LFH, lymphoid follicular hyperplasia; HU, Hounsfield unit.

Table IV. Contrast-enhanced CT attenuation of different types of soft tissue in non-thymomatous myasthenia gravis patients.

\begin{tabular}{lccc}
\hline CT attenuation $(H U)$ & LFH thymus group $(n=11)$ & Normal/involuted thymus group $(\mathrm{n}=8)$ & P-value \\
\hline Thymus region & $-25.57 \pm 58.65$ & $-117.40 \pm 6.20$ & 0.01 \\
Adipose tissue & $-105.66 \pm 16.13^{\mathrm{a}}$ & $-123.00 \pm 6.45^{\mathrm{a}}$ & 0.17 \\
Muscle tissue & $60.63 \pm 7.20^{\mathrm{a}, \mathrm{b}}$ & $57.64 \pm 3.60^{\mathrm{a}, \mathrm{b}}$ & 0.59 \\
\hline
\end{tabular}

${ }^{a} \mathrm{P}<0.001$ vs. thymus region in the same group; ${ }^{\mathrm{P}} \mathrm{P}<0.001 \mathrm{vs}$. adipose tissue in the same group. Values are expressed as the mean \pm standard deviation. LFH, lymphoid follicular hyperplasia; CT, computed tomography; HU, Hounsfield unit.

This observation coincides with the histological results that MG patients always have a thymus with atrophy and varying degrees of fat infiltration, even in LFH MG patients with B-cell lymphoid follicles and germinal centers in the thymus $(18,25)$. Regardless of whether unenhanced or contrast-enhanced CT was used, a significant difference in the mean CT attenuation was present between the LFH group and the normal/involuted thymus group $(-41.21 \pm 54.42$ vs. $-108.13 \pm 8.71$ on unenhanced CT; $-25.57 \pm 58.65$ vs. $-117.40 \pm 6.20$ on contrast-enhanced CT). Furthermore, in the LFH group, the difference in the mean unenhanced and contrast-enhanced $\mathrm{CT}$ attenuation, between the thymus region and the adipose tissue was significant, while the normal/involuted thymus group did not exhibit such a difference. This result should be interpreted with the differentiation of LFH from normal thymus in MG patients prior to thymectomy in mind. A larger study is necessary to assess more MG patients with a normal/involuted thymus to identify differences from LFH patients. Only in this way, thymectomy may be used for the patients with immune system abnormalities to cure associated diseases, including MG.

In conclusion, either unenhanced or contrast-enhanced CT may be used to distinguish LFH MG thymus from normal/involuted MG thymus, even though there were no significant differences in the dimensional measurements between the two groups.

\section{Acknowledgements}

The authors would like to thank Mr. Wang Qing, Dr Zhang Le and Mr. GuoHong at the Radiological Department of the Affiliated General Hospital of Tianjin Medical University (Tianjin, China) for their excellent technical assistance and collection of CT data. The authors also thank Mr. Wang Yuanguo, Dr Liu Yimei, Mr. Song Shihui, Mr. Zheng Kai, Mr. Wang Zhongyi and Mr. Shang Zhongliang at the Cardiac and Thoracic Surgery Department of the Affiliated General 
Hospital of Tianjin Medical University (Tianjin, China) for their advice and guidance.

\section{Funding}

No funding was received.

\section{Availability of data and materials}

The datasets used and/or analyzed during the present study are available from the corresponding author on reasonable request.

\section{Authors' contributions}

PZ performed the structural determination. TLY contributed to the conception and design. HZ collected, analyzed and interpreted the data and participated in the preparation of the manuscript. All authors read and approved the final version of the manuscript.

\section{Ethical approval and informed consent}

Written informed consent was obtained from all of the patients who participated and the Ethics Committee of the Affiliated General Hospital of Tianjin Medical University (Tianjin, China) approved the study protocols.

\section{Patient consent for publication}

Not applicable.

\section{Competing interests}

The authors declare that they have no competing interest.

\section{References}

1. Gihus NE and Verschuuren JJ: Myasthenia gravis: Subgroup classification and therapeutic strategies. Lancet Neurol 14: 1023-1036, 2015

2. Song Y, Zhou L, Miao F, Chen G, Zhu Y, Gao X, Wang Y, Pang L, Zhao C, Sun $X$ and Chen Z: Increased frequency of thymic $\mathrm{T}$ follicular helper cells in myasthenia gravis patients with thymoma. J Thorac Dis 8: 314-322, 2016.

3. Truffault F, de Montreville V, Eymard B, Sharshar T, Le Panse R and Berrih-Aknin S: Thymic germinal centers and corticosteroids in myasthenia gravis: An immunopathological study in 1035 cases and a critical review. Clin Rev Allergy Immunol 52: 108-124, 2017

4. Domeier PP, Schell SL and Rahman ZS: Spontaneous germinal centers and autoimmunity. Autoimmunity 50: 4-18, 2017.

5. Rückert JC, Ismail M, Badakhshi H, Meisel A and Swierzy M: Thymectomy in myasthenia and/or thymoma. Zentralbl Chir 139: 121-134, 2014 (In German).

6. Xie Y, Li HF, Sun L, Kusner LL, Wang S, Meng Y, Zhang X, Hong Y, Gao X, Li Y and Kaminski HJ: The role of osteopontin and its gene on glucocorticoid response in myasthenia gravis. Front Neurol 8: 230, 2017.
7. Moser B, Janik S, Schiefer AI, Müllauer L, Bekos C, Scharrer A, Mildner M, Rényi-Vámos F, Klepetko W and Ankersmit HJ: Expression of RAGE and HMGB1 in thymic epithelial tumors, thymic hyperplasia and regular thymic morphology. PLoS One 9: e94118, 2014.

8. Yablonsky P, Pischik V, Tobina MG and Atiukov M: The results of video-assisted thoracoscopic thymectomies in Saint Petersburg, Russia: 20-year of experience. J Vis Surg 3: 113, 2017.

9. Ottlakan A, Borda B, Morvay Z, Maraz A and Furak J: The effect of diagnostic imaging on surgical treatment planning in diseases of the thymus. Contrast Media Mol Imaging 2017: 9307292, 2017.

10. De Roxas RC, Bagnas MA, Baldonado JJ, Rivera JP and Roxas AA: Clinical profile and outcome of postthymectomy versus non-thymectomy myasthenia gravis patients in the Philippine general hospital: A 6-year retrospective study. Front Neurol 7: 96, 2016

11. Li HF, Hong Y, Xie Y, Hao HJ and Sun RC: Precision medicine in myasthenia graves: Begin from the data precision. Ann Transi Med 4: 106, 2016.

12. Mao ZF, Mo XA, Qin C, Lai YR and Hackett ML: Incidence of thymoma in myasthenia gravis: A systematic review. J Clin Neurol 8: 161-169, 2012

13. Araki T, Nishino M, Gao W, Dupuis J, Washko GR, Hunninghake GM, Murakami T, O'Connor GT and Hatabu H: Anterior mediastinal masses in the framingham heart study: Prevalence and CT image characteristics. Eur J Radiol Open 2: 26-31, 2015.

14. Tajima A, Pradhan I, Trucco M and Fan Y: Restoration of thymus function with bioengineered thymus organoids. Curr Stem Cell Rep 2: 128-139, 2016.

15. Nagakubo D, Krauth B and Boehm T: Genetic and non-genetic determinants of thymic epithelial cell number and function. Sci Rep 7: 10314, 2017.

16. Kranich $\mathrm{J}$ and Krautler NJ: How follicular dendritic cells shape the B-cell antigenome. Front Immunol 7: 225, 2016.

17. Mlika M, Gattoufi W, Zribi H, Braham E, Marghli A and El Mezni F: A unilocular thymic cyst associated with true thymic hyperplasia: A challenging diagnosis especially in a child. Int Med Case Rep J 8: 215-218, 2015.

18. Pereira BI and Akbar AN: Convergence of innate and adaptive immunity during human aging. Front Immunol 7: 445, 2016.

19. Sidler C, Kovalchuk O and Kovalchuk I: Epigenetic regulation of cellular senescence and aging. Front Genet 8: 138, 2017.

20. St Amour TE, Siegel MJ, Glazer HS and Nadel SN: CT appearances of the normal and abnormal thymus in childhood. J Comput Assist Tomogr 11: 645-650, 1987.

21. Francis IR, Glazer GM, Bookstein FL and Gross BH: The thymus: Reexamination of age-related changes in size and shape. AJR Am J Roentqenol 145: 249-254, 1985.

22. Nishino M, Ashiku SK, Kocher ON, Thurer RL, Boiselle PM and Hatabu H: The thymus: A comprehensive review. Radiographics 26: 335-348, 2006.

23. Manchanda S, Bhalla AS, Jana M and Gupta AK: Imaging of the pediatric thymus: Clinicoradiologic approach. World J Clin Pediatr 6: 10-23, 2017.

24. Shimamoto A, Ashizawa K, Kido Y, Hayashi H, Nagayasu T, Kawakami A, Mukae H, Hayashi T, Ohtsubo M, Shigematsu K, et al: CT and MRI findings of thymic carcinoid. Br J Radiol 90: 20150341, 2017.

25. Nishikawa N, Nagai M, Tsujii T, Kyaw WT, Tanabe N, Iwaki H, Yabe H, Ando R and Nomoto M: Treatment of myasthenia gravis in patients with elderly onset at advanced age. Jpn Clin Med 6: 9-13, 2015. 